\title{
Experimental substantiation of transdermal transport of photosensitizers by fractional laser photothermolysis(FLP)
}

\author{
Sergey Nikonov \\ Institute of Medicine and Psychology \\ Novosibirsk State University \\ Professor, the chair of Surgical \\ Diseases \\ Novosibirsk, Russia \\ sibnovomed@mail.ru
}

\author{
Danila Chernopyatov \\ Institute of Medicine and Psychology \\ Novosibirsk State University \\ Student \\ Novosibirsk, Russia \\ d9132069178@gmail.com
}

\author{
Vadim Nimaev \\ Surgical Lymphology Laboratory \\ Research Institute of Clinical and \\ Experimental Lymphology - Branch of \\ the Institute of Cytology and Genetics, \\ Siberian Branch of Russian Academy \\ of Sciences \\ Novosibirsk, Russia \\ nimaev@gmail.com
}

\begin{abstract}
- as known photodynamic therapy and photothermolysis are used to treat skin diseases and to correct signs of aging. These methods are minimally invasive but their disadvantages are related to skin damage, prolonged phototoxicity with intravenous photosensitizer, and the insufficient penetration of the drug through the skin by local application. The possibility of minimally invasive transdermal drug transport by using photothermolysis and photodynamic therapy technologies on the example of the delivery of the Radagel photosensitizer has been demonstrated. The method of transdermal delivery of a photosensitizer through the skin micropores, which are formed by focused energy rays of a nonablative laser has been developed for the side effects prevention of laser techniques with the summation of their advantages.
\end{abstract}

Keywords - photodynamic therapy; photosensitivity; photothermolysis; laser energy; skin disease;transdermal transport

\section{BACKGROUND}

In the treatment of benign, precancerous and malignant skin diseases, the technology of photodynamic therapy (PDT) is actively introduced, which based on the selective damage of a pathological substrate by a drug photosensitizer (PS) in combination with electromagnetic radiation of the visible red range in the presence of tissue oxygen. The traditional method of intravenous photosensitization of the body involves the introduction of high doses of expensive PS (Radachlorin, Photoditazin, Verteporfin, Alasens), which is associated with long-term retention of drugs in healthy and pathologically altered cells, which leads the for patients to observe the light protection regimen in the post-treatment period to avoid skin burns, photodermatitis, prolonged erythema and phototoxic face edema. In this regard, for the necessities of outpatient dermatology, dermato-oncology and cosmetology, the local application photosensitization of pathological changes by gel forms of FS with subsequent PDT light energy sources is the most acceptable. Despite the fact that when applied to the skin, PS shows a tropism to the tumor, inflamed, and dystrophic cells, the distribution of the drug in the layers of the dermis and the depth of its transdermal penetration is unknown.

We have developed a targeted local photosensitization of the deep layers method of the skin by fractional laser photothermolysis using focused pulsed-periodic radiation from a fiber laser. Microthermal zones which were formed on the skin of experimental mice have provided the efficient transdermal transport of FS, which was proved by pathomorphological studies of skin layers with confocal microscopy. Based on the fluorescence characteristic of PS, the distribution from the epidermis through the stratum corneum, dermis to subcutaneous adipose tissue was shown. All in all, the FLP method for local PS transdermal transport can be highly effective in outpatient PDT in dermatology and cosmetology.

\section{PURPOSE}

To substantiate experimentally the applicability of photodynamic fractional laser photothermolysis (PFLP) for creation new treatment technologies in dermatocosmetology and dermato-oncology.

\section{WORK TASKS}

To study the transport possibilities of photothermolysis for transdermal delivery of a photosensitizer to the laboratory animals skin.

To study the range of application of "Chlorin e6" fluorescence effects for evidance the usefulness of its transdermal transport through non-ablative laser photothermolysis.

\section{METHODS AND MATERIALS}

In the work, 3-month-old male mice were used. They were divided into four groups:

The first group (16 individuals) - fractional laser photothermolysis (FLP) of the skin of the abdominal wall with a "Lakhta Milon" semiconductor laser $(\lambda=970 \mathrm{~nm})$ with an average power of $4 \mathrm{~W}$ in a scanning pulse-periodic mode $(\mathrm{t}=$ $10 \mathrm{~ms} / 10 \mathrm{~ms}$ ) through a quartz-polymer fiber with a focuser to form the epidermis of microthermal zones (MTZ) with a density of $16 \mathrm{MTZ} / \mathrm{cm}^{2}$ with a dose density of energy in the pulse of $0.25 \mathrm{~J} / \mathrm{mm}^{2}$

Thus, 16 microthermal zones (MTZ) with a diameter of $0.4 \mathrm{~mm}$ are formed in the epidermis over an area of $1 \mathrm{sq} \mathrm{cm}$.

The second group (16 individuals) - is performed by FLP, after which the photosensitizer Radagel $\{0.5 \%-0.1 \mathrm{~g}(\mathrm{n}=10)\}$ is applied to the skin for 20 minutes. Then, a "Lakhta Milon" semiconductor laser $(\lambda=662 \mathrm{~nm})$ is irradiated with an average power of $2 \mathrm{~W}$ in a pulse-periodic mode $(\mathrm{t}=100 / 100 \mathrm{~ms})$ through an optical fiber with a light spot size of $1,2 \mathrm{~mm}^{2}$ on the skin.

The third group (16 individuals) - the photosensitizer Radagel $\{0.5 \%-1 \mathrm{~g}(\mathrm{n}=10)\}$ is applied to the skin of the animal for 20 minutes. Then, a "Lakhta Milon" semiconductor 
laser $(\lambda=662 \mathrm{~nm})$ is irradiated with an average power of $2 \mathrm{~W}$ in a pulse-periodic mode $(\mathrm{t}=100 / 100 \mathrm{~ms})$ through an optical fiber with a light spot size on the skin of $1,2 \mathrm{~mm}^{2}$

The fourth group (4 individuals) - control without exposure.

After the completion of laser irradiation, one individual from every experimental group undergoes intravital fluorescence diagnostics in the blue spectrum of $406 \mathrm{~nm}$ and in the red spectrum of $630 \mathrm{~nm}$ on a "Fluovisor" instrument.

Then the animals were euthanized by the method of cervical vertebrae dislocation, the anterior abdominal wall was dissected, 4 skin flaps were taken, one was sent for electron microscopy, one for histological examination, and transverse sections was made from two flaps using a microtome (cryotome) for subsequent confocal laser microscopy LSM 510 scanning microscope to record Radagel fluorescence effects.

A similar sequence of studies was carried out on the 7 th, 14th and 28th days.

It complies with the provisions of the Federal Law on Amendments to Article 245 "Cruelty to Animals." Entered into force on December 20, 2017. Russia.

\section{RESULTS}

On the results of fluorescence diagnostics, in conclusion we can say that the highest fluorescence of the Radagel preparation was recorded in the skin after fractional photothermolysis followed by application photosensitization, which was indicated a pronounced intradermal accumulation of the photosensitizer in comparison with the usual application to the skin.

Laser confocal microscopy was carried out at the optimal wavelengths for investigation: excitation of fluorescence at $458 \mathrm{~nm}$, registration of fluorescence at $675 \mathrm{~nm}$.
In reseach of an individual with FLP, no changes in the structure of the skin were detected, except for light loosening of collagen fibers. Also, the absence of autofluorescence of skin structures is diagnostically important.

According to the results individuals from the group prepared for PFLP, it can be seen that the largest part of the photosensitizer accumulated in the epithelium and papillary layer of the skin, but the intense fluorescence recorded by us in collagen fibers, skin structures (hair and sebaceous follicles), hypodermis and muscle fibers indicates that preparation of the skin by fractional laser photothermolysis creates the conditions for active transdermal transport of the photosensitizer. In the traditional application the drug Radagel on the skin surface, its penetrating ability is limited by the epithelial layer, which implies that the induced photodynamic reaction will have exclusively surface effects.

\section{CONCLUSION}

The idea of controlled transdermal photosensitizer transport for the implementation of medical technology of photodynamic fractional laser photothermolysis was theoretically substantiated.

For the first time in the conditions of an experiment, the possibility of creating transport channels in the skin for intraand transdermal transport of drugs was successfully demonstrated using the drug photosensitizer Radagel.

The ability of non-invasive fractional laser photothermolysis to provide conditions for efficient transdermal transport of the photosensitizer to all skin layers during application is proved experimentally.

This option of local photosensitization of the skin is manageable and, depending on the dose of the photosensitizer used, can be used in cosmetology, dermatology, gynecology, oncology. 